\title{
Assessment of Risks in Management Factors
}

\author{
Dong Ju Kim¹), P. Lakshmi Manjusha2)
}

\begin{abstract}
Software risk management are often outlined as a trial to formalize risk related to overall size of the software system to be engineered or changed and therefore the constraints obligatory by management with the degree to that the method outlined and is developed. it's without delay applicable to a collection of principles/practices and done by employing a survey instrument to research this claim additional. This survey ends up in hope for development of the system. With the assistance of proof given by this paper, we will grasp the categories of positive responses. we'd like to look at the failure to satisfy the necessity would degrade the performance surroundings is related with in management of those elements. If any risk i.e., minor delays in software system modifications, operational delays and the way the environmental problems and therefore the risk management will influence the software system risk. During this paper we tend to investigate additional impacts of risk management practices on software system development. We tend to square measure getting in a broad and careful perspective on software system risk .The list is additionally accepted and had been wide applied in observe to search out risk management plans. 2 risk management factors and 4 environmental factors mitigate demand management risks. It's fascinating to look at the risks during which necessities management is known together of the foremost advanced drawback in software system development. The survey instrument used is unidirectional analysis of variance to try and do multiple comparisons to look at however risk management and environmental factors influence every risk obtained by the code. The 2 risk management factors square measure risk identification and risk analysis and therefore the four environmental factors square measure planning risks, demand management risks.
\end{abstract}

Keywords: analysis, comparisons, fascinating, investigate, perspective.

\section{INTRODUCTION}

Risk identification is that the method of deciding risks that would doubtless stop the program, enterprise, or investment from achieving its objectives. It includes documenting and human action the priority[1]:

Received(February 12, 2015), Review Result(1st: February 27, 2015, 2nd: March 9, 2015), Accepted(May 10, 2015)

${ }^{1}$ Chung-Ang University, \#301-116, 221, Huksok-dong, DongJak-Gu Seoul, Rep. of Korea email: dongju@naver.com

${ }^{2}$ (Corresponding Author) Vetri Vikas Educational Institutions, Mallur (Via), Rasipuram Taluk, Annamalaipatti, Tamil Nadu, India email: lakshmimanjusha16@gmail.com 
- Identify the project, product and business risk.

- Check whether or not the chance may be a technological risk, folks risk, demand risk and estimation risks.

Risk identification may be a systematic commit to specify threats to the project set up. By characteristic famous and predictable risks, the project manager takes a primary step toward avoiding them once potential and dominant them once necessary:

- Generic risks

- Risks that square measure a possible threat to each software system project

- Product-specific risks

Risks that may be known solely by those a with a transparent understanding of the technology, the people, and therefore the surroundings that's specific to the software system that's to be engineered. This needs examination of the project set up and therefore the statement of scope "What special characteristics of this product might threaten our project plan?"

Some common ways of risk identification are:

- Taxonomy primarily based Risk Identification: The potential risk sources square measure bust down, therefore taxonomy. A form is created best on existent knowledge; the answers to the queries square measure the chance.

- Objective primarily based Risk Identification: a company or any endeavour incorporates a sure objective/s. Any activity that's deemed associate obstacle within the action of a similar is perceived as risk.

- Scenario primarily based Risk Identification: Here numerous eventualities, which can be alternative routes to attain associate objective, square measure created. If associate unwanted situation is formed, a threat is perceived with a similar.

- Common Risk Check: There square measure sure risks that square measure common to associate trade. every risk is listed and checked on time.

\subsection{Identification of root causes:}

Program offices ought to examine their programs associated determine root causes by 
reducing program components to grade of detail that allows an judge to know the importance of any risk and determine its causes. this is often a sensible manner of addressing the big and various range of risks that usually occur in any software system organisation[2-3].

Typical risk sources include threat. The sensitivity of the program to uncertainty within the threat description, the degree to that the system style would have to be compelled to amendment if the threat's parameters amendment, or the vulnerability of the program to foreign intelligence assortment efforts (sensitivity to threat countermeasure).

- Requirements. The sensitivity of the program to uncertainty within the system description and necessities, excluding those caused by threat uncertainty. necessities embody operational desires, attributes, performance and readiness parameters (including KPPs), constraints, technology, style processes, and WBS parts.

- Technical Baseline. the flexibility of the system configuration to attain the program's engineering objectives supported the out there technology, style tools, style maturity, etc. Program uncertainties and therefore the processes related to the "ilities" (reliability, supportability, maintainability, etc.) should be thought-about. The system configuration is associate degree agreed-to description (an approved and discharged document or a collection of documents) of the attributes of a product, at a degree in time, that is a basis for outlining amendment.

- Test and analysis. The adequacy and capability of the take a look at and analysis program to assess attainment of great performance specifications and verify whether or not the system is operationally effective, operationally appropriate, and practical.

- Modeling and Simulation (M\&amp;S). The adequacy and capability of M\&amp;S to support all life-cycle phases of a program victimization verified, validated, and licensed models and simulations.

- Technology. The degree to that the technology planned for the program has incontestible enough maturity to be realistically capable of meeting all of the program's objectives.

- Logistics. the flexibility of the system configuration and associated documentation to attain the program's supplying objectives supported the system style, maintenance conception, network style, and handiness of support information and resources.

- Production/Facilities. the flexibility of the system configuration to attain the program's production objectives supported the system style, producing processes chosen, and handiness of producing resources (repair resources within the sustenance phase).

- Concurrency. The sensitivity of the program to uncertainty ensuing from the combining or overlapping of life-cycle phases or activities. 
- Industrial Capabilities. the talents, experience, resources, and data of the contractors to style, develop, manufacture, and support the system.

- Cost. the flexibility of the system to attain the program's life-cycle support objectives. This includes the results of budget and affordability selections and therefore the effects of inherent errors within the cost estimating technique(s) used (given that the technical necessities were properly outlined and taking under consideration best-known and unknown program information).

- Management. The degree to that program plans and methods exist and area unit realistic and consistent. The government's acquisition and support team ought to be qualified and sufficiently staffed to manage the program.

- Schedule. The sufficiency of the time allotted for playacting the outlined acquisition tasks. This issue includes the results of programmatic schedule selections, the inherent errors in schedule estimating, and external physical constraints.

- External Factors. the provision of state resources external to the program workplace that area unit needed to support the program like facilities, resources, personnel, government provided with instrumentality, etc.

- Budget. The sensitivity of the program to budget variations and reductions and therefore the resultant program turbulence.

- Earned worth Management System. The adequacy of the contractor's EVM method and therefore the realism of the integrated baseline for managing the program.

\subsection{Risk Analysis}

Definition: Risk analysis is that the method that permits management to demonstrate that it's met its obligation of due diligence once creating a call concerning moving forward with a replacement project, cost, investment strategy, or alternative such business method[4].

- Assess the chance and consequences of those risks that area unit known within the on top of section.

- Assessing the chance and occurrences of every and risk known as a result of typically the chance could also be low, high, moderate and really high.

The intent of risk analysis is to answer the question "How massive is that the risk?" by:

- Considering the chance of the basis cause occurrence;

- Identifying the doable consequences in terms of performance, schedule, and cost; and

- Identifying the danger level victimization the danger news Matrix shown in Figure. 


\subsection{Description of risk news matrix}

Each undesirable event that may have an effect on the success of the program (performance, schedule, and cost) ought to be known and assessed on the chance and consequence of prevalence[5]. a typical format for analysis and news of program risk assessment findings facilitates common understanding of program risks the least bit levels of management. the danger news Matrix below is usually wont to verify the extent of risks known among a program. the extent of risk for every root cause is reportable as low (green), moderate (yellow), or high (red).

The level of chance of every root cause is established utilizing mere criteria for instance, if the basis cause has associate degree calculable fifty p.c chance of occurring, the corresponding chances are level three.

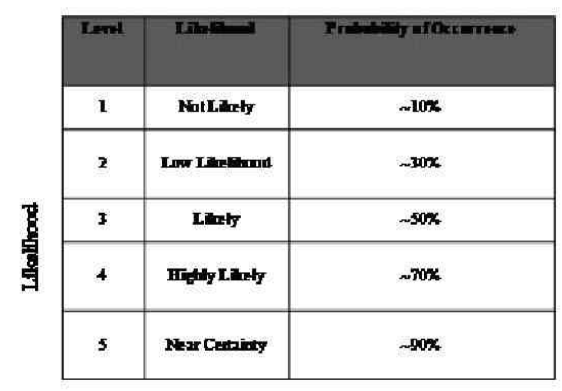

[Fig. 1] The level of chance of every root

The level and types of consequences of each risk are established utilizing criteria such as those described. A single consequence scale is not appropriate for all programs, however. Continuing with the prior example of a root cause with a 50 percent probability of occurring, if that same root cause has no impact on performance or cost, but may likely result in a minor schedule slippage that won't impact a key milestone, then the corresponding consequence is a Level 3 for this risk. For clarity it is also classified as a schedule risk since its root cause is schedule related.

\subsection{Risk Monitoring}

In order to monitor the current status of the risks and the measures defined, metrics can be Specified for the risks. These metrics can be quantitative, such as the probability of occurrence 
or effort for and cost of control But they can also be qualitative, e.g. an appraisal of project staff's motivation. Another monitoring method is to use triggers, which are threshold values for metrics which Trigger measures when reached.

\subsection{Risk planning}

The intent of risk planning is to answer the question "What is the program approach for addressing this potential unfavourable consequence?" One or more of these mitigation options may apply:

- Avoiding risk by eliminating the root cause and/or the consequence,

- Controlling the cause or consequence,

- Transferring the risk, and/or

- Assuming the level of risk and continuing on the current program plan.

- Risk planning is the activity that identifies, evaluates, and selects options to set risk at acceptable levels given program constraints and objectives.

- Risk planning is intended to enable program success. It includes the specifics of what should be done, when it should be accomplished, who is responsible, and the funding required to implement the risk plan.

- The most appropriate program approach is selected from the mitigation options listed above and documented in a risk mitigation plan.

\section{Risk management analysis}

\subsection{Risk management}

A set of principles and practices to identify, analyse and handling risk factors to improve the chances of achieving a successful project outcome(or) to avoid project failure. It's a process to identify where the risk have occurred and setting up strategy's to deal with it by controlling[6-8]. It's about making evaluation to the true level of risk in a realistic manner.

- Anticipate and identify risk: To perform any action on the risk occurred firstly the module where the risk have occurred must be identified. 
- Minimize the impact or damage or loss: After identifying the risk necessary steps are to be taken so that the damage that can be caused to the project can be minimized so that risk cannot abort the entire functionality.

- Reduce the Probability: Risk cannot be completely reduced but by taking some necessary steps the risk can be reduced so that the risk occurred becomes negligible.

- Ensure management awareness of risk: All the project members must have awareness of the possible risk and how to manage them so that risk identification is a easy task to be done by all the project members.

We have two types of analyses. They are 'Qualitative analysis' and 'Quantitative analysis'.

Here, we considered Qualitative analysis and considered different tree analyses.

Risks to staff and organizations are prevalent in business system. Thus, it is necessary for an organization to have qualified business risk managers to assess, develop, implement, and monitor risk management plans with the goal of minimizing exposure. There are many priorities to a software organization, such as finance, safety and most importantly in any software system.

In any business systems, the first step is to assess and identify the potential risks that are creating problems to our business.

- While assessing any business, we should identify the types of risks.

- The risks may be internal and external to any business.

- These risks may be directly or indirectly affect the business.

- Risks in a business may be hazard -based (or) uncertainty - based

\subsection{Direct risks to any business system}

Some of the common categories of risk are:

- natural disasters

- environmental

- property and equipment

- security

- utilities and services

These square measure external to our business. 


\subsection{Indirect risks}

These varieties of risks won't be able to acknowledge directly and that they impact our business internally. These square measure unpredictable risks and that we cannot determine them earlier. When an extended time, the project might; fail thanks to these varieties of indirect risks and unknown reasons.

\subsection{Managing business risks}

By understanding potential risks to your business and finding ways in which to minimise their impacts, by serving to your business to recover quickly if an event happens. Types of risk vary from business to business, however making ready a risk management set up involves a typical method to manage any varieties of risks in a very business system. For managing risks, we must always prepare a risk management set up by aggregation all the knowledge associated with risks i.e., all the resources, budget.

\subsection{Theoretical analysis}

Probability analysis:

- Probability analysis overcomes the constraints of sensitivity analysis by specifying a likelihood distribution for every variable, and so considering things wherever any or all of those variables are often modified at constant time.

- Defining the likelihood of incidence of any specific variable could also be quite troublesome, significantly as political or business environments will amendment quite speedily.

- As with sensitivity analysis, the vary of variation is subjective, however ranges for several time and value parts of a project estimate ought to be inclined toward overrun, thanks to the natural optimism or omission of the figurer 2.

- Low impact/ow probability: Risks in the bottom left corner are low level, and ignore them.

- Low impact/high probability: Risks in the top left corner are of moderate important if these happen, they cope with them and move on. However, they reduce the likelihood that they occur. 
- High impact/low probability: Risks in the bottom right corner are of highly important if they do occur, but they are very unlikely to happen. For these, however, we should do what you can to reduce the impact that they have if they do occur, and you should have contingency plans.

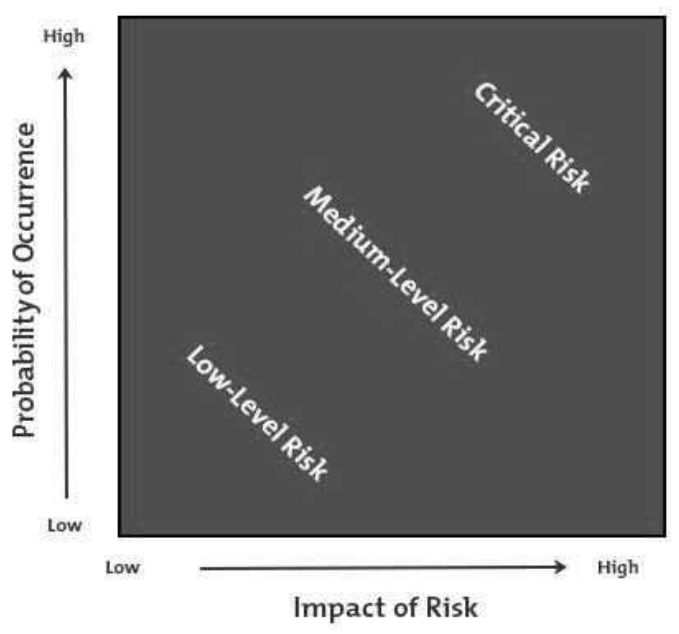

[Fig. 2] Level of Risk

\section{CONCLUSION}

We consider different types of Tree Analyses and come up with a new approach to overcome the impacts of risk and overcome the risk that occur in the Business system. Risk management is a very essential concept in a project development. There are few companies that allot managers especially to deal with the risks. They need to pre assess the risk, try to solve them in all the ways possible. Risk is not a flaw for the project but it makes the project deviate from its normal functionality. If all the risks are identified then it can be considered as a way to develop a project which is perfect and have great durability to sustain in this software field as in today's generation day-to-day new technologies emerge. So, A project that is developed and have undergone risks and finally managed can be regarded as a best project. 


\section{References}

[1] T. Thüma, C. Kästnerb, F. Benduhna, J. Meinickea, G. Saakea and T. Leichc, FeatureIDE: An extensible framework for feature-oriented software development, Science of Computer Programming, (2012), Vol.79, No.1, pp.70-85.

[2] A. Arcuri and L. Briand, A Hitchhiker's guide to statistical tests for assessing randomized algorithms in software engineering, Journal of Software: Testing, Verification and Reliability, (2014), Vol.24, No.3, pp.219-250.

[3] https://certifedpmp.wordpress.com/2008/09/17/risk-analysis-techniques/, December 11 (2014).

[4] https://www.business.qld.gov.au/business/running/risk-management/identifying-business-risk, May 1 (2014).

[5] https://www.business.qld.gov.au/business/running/risk-management/risk-management-plan-business-impactanalysis/identify-risk-business, July 25 (2014).

[6] K. J. Hole, A. N. Kingsheim, L. H. Netland, Y. Espelid, T. Tjostheim, and V. Moen, Risk Assessment of a National Security Infrastructure, IEEE Security \& Privacy, (2009), Vol.7, No.1, pp.34-41.

[7] C. Nan, I. Eusgeld, Adopting HLA standard for interdependency study, Resilience Engineering and System Safety, (2011), Vol.96, No.1, pp.149-159.

[8] http://www.bmi.bund.de, March 2 (2012). 